\title{
Self-similar cosmological solutions with dark energy. I: formulation and asymptotic analysis
}

\author{
${ }^{1}$ Tomohiro Harada* ${ }^{1,2,3,4}$ Hideki Maeda ${ }^{\dagger}$ and ${ }^{5,6}$ B. J. Carr ${ }^{\ddagger}$ \\ ${ }^{1}$ Department of Physics, Rikkyo University, Toshima, Tokyo 171-8501, Japan \\ ${ }^{2}$ Centro de Estudios Científicos (CECS), Arturo Prat 514, Valdivia, Chile \\ ${ }^{3}$ Department of Physics, International Christian University, \\ 3-10-2 Osawa, Mitaka-shi, Tokyo 181-8585, Japan \\ ${ }^{4}$ Graduate School of Science and Engineering, Waseda University, Tokyo 169-8555, Japan \\ ${ }^{5}$ Astronomy Unit, Queen Mary, University of London, Mile End Road, London E1 4NS, UK \\ ${ }^{6}$ Research Center for the Early Universe, Graduate School of Science, University of Tokyo, Tokyo 113-0033, Japan
}

(Dated: November 3, 2018)

\begin{abstract}
Based on the asymptotic analysis of ordinary differential equations, we classify all spherically symmetric self-similar solutions to the Einstein equations which are asymptotically Friedmann at large distances and contain a perfect fluid with equation of state $p=(\gamma-1) \mu$ with $0<\gamma<$ $2 / 3$. This corresponds to a "dark energy" fluid and the Friedmann solution is accelerated in this case due to anti-gravity. This extends the previous analysis of spherically symmetric self-similar solutions for fluids with positive pressure $(\gamma>1)$. However, in the latter case there is an additional parameter associated with the weak discontinuity at the sonic point and the solutions are only asymptotically "quasi-Friedmann", in the sense that they exhibit an angle deficit at large distances. In the $0<\gamma<2 / 3$ case, there is no sonic point and there exists a one-parameter family of solutions which are genuinely asymptotically Friedmann at large distances. We find eight classes of asymptotic behavior: Friedmann or quasi-Friedmann or quasi-static or constant-velocity at large distances, quasi-Friedmann or positive-mass singular or negative-mass singular at small distances, and quasi-Kantowski-Sachs at intermediate distances. The self-similar asymptotically quasi-static and quasi-Kantowski-Sachs solutions are analytically extendible and of great cosmological interest. We also investigate their conformal diagrams. The results of the present analysis are utilized in an accompanying paper to obtain and physically interpret numerical solutions.
\end{abstract}

PACS numbers: 04.70.Bw, 95.36.+x, 97.60.Lf, 04.40.Nr, 04.25.Dm

\section{INTRODUCTION}

There is great interest in spherically symmetric selfsimilar solutions to Einstein's equations because of their numerous applications in astrophysics and cosmology [1]. Indeed there is now considerable evidence for the "similarity hypothesis", which postulates that spherically symmetric solutions may naturally evolve to self-similar form in a variety of situations. The status of this hypothesis has been recently reviewed by Carr and Coley [2]. In view of this, it is clearly important to have as complete a classification of spherically symmetric self-similar solutions as possible.

Spherically symmetric self-similar solutions have the feature that all dimensionless quantities can be expressed in terms of $z=r / t$, where $r$ and $t$ are suitably chosen radial and time coordinates. If the source of the gravitational field is a single perfect fluid, Cahill and Taub [3] have shown that the only barotropic equation of state compatible with the similarity assumption has the form $p=(\gamma-1) \mu$ for some constant $\gamma$, where $\mu$ and $p$ are the energy density and the pressure, respectively. In this

\footnotetext{
*Electronic address:harada@rikkyo.ac.jp

$\dagger$ Electronic address:hideki@cecs.cl

${ }_{\ddagger}^{\ddagger}$ Electronic address:B.J.Carr@qmul.ac.uk
}

case, for a given value of $\gamma$, the solutions are described by two parameters and, providing one restricts attention to shock-free perfect fluids with positive pressure $(1<\gamma \leq 2)$, they have been completely classified. This classification has been achieved using a combination of the "comoving" approach (in which the coordinates are adapted to the fluid 4-velocity) and the "homothetic" approach (in which the coordinates are adapted to the homothetic vector). These approaches have been used by Carr and Coley [4] (henceforth CC) and Goliath et al. 5., respectively, although a full understanding of the solutions requires that one combines them [6].

A key step in the $\mathrm{CC}$ analysis is the derivation of all possible asymptotic behaviors at large and small distances [7]. For positive pressure, there are at least three kinds of behavior at large spatial distances (usually corresponding to the limit $z \rightarrow \infty$ ): (1) asymptotically Friedmann (1-parameter); (2) asymptotically KantowskiSachs (1-parameter), though these are probably unphysical for $\gamma>2 / 3$ ); and (3) asymptotically quasi-static (2-parameter). There are also two families of solutions which exist only when $\gamma>6 / 5$ : (4) asymptotically Minkowski at infinite $z$ (1-parameter); and (5) asymptotically Minkowski at finite $z$ but infinite physical distance (2-parameter). At small spatial distances, the solutions are of four kinds: they contain either (a) a black hole singularity or (b) a naked singularity at finite $z$ (but zero physical distance) or they can be connected to $z=0$ via 
a sonic point, in which case they are either (c) static or (d) represent a perturbation of the Friedmann solution.

The complete family of $1<\gamma \leq 2$ solutions can now be found by combining the five kinds of large-distance behavior and four kinds of small-distance behavior. The way in which one connects the large-distance and smalldistance solutions depends crucially on whether or not the solution passes through a sonic point. If the solutions remain supersonic (or subsonic) everywhere, then the small- $z$ behavior is uniquely determined by the large$z$ behavior. However, if there is a sonic point, the behavior of the solutions is much more complicated because the equations do not determine their behavior uniquely there, so there can be a discontinuity. Indeed, only a subset of solutions are "regular" at a sonic point in the sense that the pressure gradient is finite and they can be extended beyond it. Because of this feature, the family of solutions with a regular center and a regular sonic point have a band structure, with the solutions which are analytic at the center and sonic point forming a discrete subset of these [8, 9, 10]. This feature is very important for naked singularity formation and critical behavior in the gravitational collapse of a perfect fluid 11, 12].

In this and the accompanying papers [13] (henceforth Paper II), we take a first step in extending the classification of CC to the negative pressure case $(\gamma<1)$, using a combination of numerical and analytical studies. In fact, part of the work is already done, because ref. [7] does include the asymptotic analysis for $\gamma<1$. However, there are some errors in that work (see Appendix A) and the full family of solutions has not yet been analyzed. This paper will focus particularly on the case with $0<\gamma<2 / 3$. Although this equation of state violates the strong energy condition and was little emphasized by $\mathrm{CC}$, it may be very relevant for cosmology - both in the early universe (when inflation occurred [14]) and at the current epoch [15] (when acceleration may be driven by some form of "dark energy"). In both situations the matter model exhibits "anti-gravity" but the dominant, null and weak energy conditions still hold.

As in the positive pressure case, it should be stressed that our classification does not cover imperfect or multiple fluids or solutions with shocks. The accelerated expansion of the universe can also be realized by a scalar field with a flat potential (i.e. quintessence). The nonexistence of self-similar black hole solutions embedded in an exact Friedmann universe for this case was already demonstrated in ref. [16] and this is clearly complementary to the present work.

There are several key differences between the $0<\gamma<$ $2 / 3$ and $2 / 3<\gamma<2$ cases. A purely formal difference is that the limiting values of $z$ for large and small spatial distances in the flat Friedmann solution are reversed: large spatial distances now correspond to $z \rightarrow 0$ and small ones to $z \rightarrow \infty$. The other differences have more physical significance. The first is that there are no soundwaves, since the sound-speed $c_{s}=\sqrt{d p / d \mu}=c \sqrt{\gamma-1}$ is imaginary. This considerably simplifies the analysis, since there can be no discontinuities and solutions are analytic everywhere. The second is that there is no exact static solution. although there are still asymptotically quasi-static solutions (a point which was missed in ref. [7]). On the other hand, the Kantowski-Sachs and asymptotically quasi-Kantowski-Sachs solutions now become physical. Finally, the solutions which were asymptotically Minkowski at large distances are now replaced with solutions which are asymptotically singular at small distances.

Despite these differences, many features of the CC classification still apply. In particular, there is still a 1-parameter family of solutions asymptotic to the flat Friedmann model at large distances and these are of great physical interest since they may have cosmological applications. In the positive-pressure case, some of these solutions are supersonic everywhere and contain black holes which grow at the same rate as the particle horizon [17, 18]. Others represent density perturbations in a Friedmann background which always maintain the same form relative to the particle horizon 19]. However, recently it was pointed out that none of these positivepressure solutions are "properly" asymptotic Friedmann because they exhibit a solid angle deficit at infinity [20]. They may still be relevant to the real universe (since observations may not preclude such an angle deficit) but it would be more accurate to describe them as "quasiFriedmann".

In the $0<\gamma<2 / 3$ case, we will show that there are genuine asymptotically Friedmann solutions. We will analyze these solutions numerically and exploit these results in Paper II [13] to interpret the solutions physically . The key feature is the existence of asymptotically KantowskiSachs and static solutions, both of which are extendible analytically. This leads to the possibility of cosmological black hole, wormhole and white hole solutions.

The plan of this paper is as follows. In Section II, we present the basic field equations for spherically symmetric self-similar spacetimes. We describe some exact solutions in Section III and solutions which are asymptotic to these In Section IV. We summarize our results and discuss their implications in Section V.

\section{BASIC EQUATIONS}

We consider a spherically symmetric spacetime with the line element

$$
d s^{2}=-e^{2 \Phi(t, r)} d t^{2}+e^{2 \Psi(t, r)} d r^{2}+R(t, r)^{2} d \Omega^{2},
$$

where $d \Omega^{2} \equiv d \theta^{2}+\sin ^{2} \theta d \varphi^{2}$. We take the matter field to be a perfect fluid with energy-momentum tensor

$$
T_{\mu \nu}=p g_{\mu \nu}+(\mu+p) u_{\mu} u_{\nu}
$$

where $p$ and $\mu$ are the pressure and the energy density, respectively, $u_{\mu}$ is the 4 -velocity of the fluid, and we use 
units with $c=1$. We will adopt comoving coordinates, so that the 4 -velocity is

$$
u^{\mu} \frac{\partial}{\partial x^{\mu}}=e^{-\Phi} \frac{\partial}{\partial t} .
$$

The field equations can then be written in the following form:

$$
\begin{aligned}
p_{, r} & =-(\mu+p) \Phi_{, r}, \\
\mu_{, t} & =-(\mu+p)\left(\Psi_{, t}+2 \frac{R_{, t}}{R}\right) \\
m_{, r} & =4 \pi \mu R_{, r} R^{2} \\
m_{, t} & =-4 \pi p R_{, t} R^{2} \\
0 & =-R_{, r t}+\Phi_{, r} R_{, t}+\Psi_{, t} R_{, r}, \\
m & =\frac{R}{2 G}\left(1+e^{-2 \Phi} R_{, t}^{2}-e^{-2 \Psi} R_{, r}^{2}\right),
\end{aligned}
$$

where a comma denotes partial differentiation and $G$ is the gravitational constant. The first two equations correspond to the energy-momentum conservation and the next two specify the Misner-Sharp mass $m$. Five of the above six equations are independent. Throughout this paper, we call the direction of increasing (decreasing) $t$ the future (past) direction.

A spacetime is self-similar if it admits a homothetic Killing vector $\xi^{\mu}$, which is defined by

$$
\mathcal{L}_{\xi} g_{\mu \nu}=2 g_{\mu \nu}
$$

where $\mathcal{L}_{\xi}$ denotes the Lie derivative along $\xi^{\mu}$. Cahill and Taub [3] first investigated spherically symmetric selfsimilar solutions in which the homothetic Killing vector is neither parallel nor orthogonal to the fluid flow vector. They showed that - by a suitable coordinate transformation - such solutions can be put into a form in which all dimensionless quantities are functions only of the dimensionless variable $z \equiv r / t$. In this case, $\xi^{\mu}$ is given by

$$
\xi^{\mu} \frac{\partial}{\partial x^{\mu}}=t \frac{\partial}{\partial t}+r \frac{\partial}{\partial r} .
$$

The line element in a spherically symmetric self-similar spacetime can be written as

$$
d s^{2}=-e^{2 \Phi(z)} d t^{2}+e^{2 \Psi(z)} d r^{2}+r^{2} S^{2}(z) d \Omega^{2} .
$$

A hypersurface $\Sigma$ of constant $z$ is called a similarity surface and is generated by the homothetic Killing vector. The induced metric on $\Sigma$ is

$$
d s_{\Sigma}^{2}=-\left(1-V^{2}\right) e^{2 \Phi} d t^{2}+r^{2} S^{2} d \Omega^{2}
$$

where $V \equiv|z| e^{\Psi-\Phi}$ is the speed of the fluid relative to the surfaces of constant $z$. The area radius $R=r S$ must be positive but $S$ and $r$ need not be. (Note that CC adopt a convention in which $r$ is always positive.) A similarity surface is spacelike for $\left(V^{2}-1\right) e^{2 \Phi}>0$, timelike for $\left(V^{2}-1\right) e^{2 \Phi}<0$ and null for $\left(1-V^{2}\right) e^{2 \Phi}=0$. In the latter case, $\xi^{\mu}$ is also null and the similarity surface is called a similarity horizon.

It is helpful to rewrite Eq. (2.12) in the conformally static form:

$$
\begin{aligned}
d s^{2}= & -e^{2 \tau}\left[e^{2 \Phi}\left\{(1+V) d \tau+\frac{V}{z} d z\right\}\left\{(1-V) d \tau-\frac{V}{z} d z\right\}\right. \\
& \left.+z^{2} S^{2} d \Omega^{2}\right]
\end{aligned}
$$

where the conformal factor depends only on $\tau \equiv \ln |t|$. To obtain the causal structure of the self-similar spacetime, we can then use the analogy with the static spacetime. This is because the homothetic Killing vector, similarity surfaces and similarity horizons are the counterparts of the Killing vector, Killing surfaces and Killing horizons in the static case (see e.g. ref. [22]).

The Einstein equations imply that $p, \mu$ and $m$ must have the form

$$
\begin{aligned}
8 \pi G \mu & =\frac{W(z)}{r^{2}}, \\
8 \pi G p & =\frac{P(z)}{r^{2}}, \\
2 G m & =r M(z),
\end{aligned}
$$

where we assume that the energy density is non-negative $(W \geq 0)$. Then the Einstein and energy-momentum conservation equations reduce to ordinary differential equations for the non-dimensional functions with respect to the self-similar variable $z$ :

$$
\begin{aligned}
-2 P+P^{\prime} & =-(W+P) \Phi^{\prime} \\
-W^{\prime} & =(W+P)\left(\Psi^{\prime}+2 \frac{S^{\prime}}{S}\right) \\
M+M^{\prime} & =W S^{2}\left(S+S^{\prime}\right) \\
-M^{\prime} & =P S^{2} S^{\prime} \\
0 & =S^{\prime \prime}+S^{\prime}-\Phi^{\prime} S^{\prime}-\Psi^{\prime}\left(S+S^{\prime}\right) \\
M & =S\left[1+z^{2} e^{-2 \Phi} S^{\prime 2}-e^{-2 \Psi}\left(S+S^{\prime}\right)\right.
\end{aligned}
$$

where a prime denotes a derivative with respect to $\ln |z|$.

We assume the equation of state has the form $p=$ $(\gamma-1) \mu$, which is the only barotropic one compatible with the homothetic assumption. The dominant energy condition requires $0 \leq \gamma \leq 2$. In this paper we exclude the value of 1 (corresponding to dust) since this needs special treatment and has been analyzed in ref. 23]. We also exclude $\gamma=0$, corresponding to a cosmological constant, since this is incompatible with self-similarity. Equations (2.18) and (2.19) can then be integrated to give

$$
\begin{aligned}
e^{\Phi} & =c_{0} z^{2(\gamma-1) / \gamma} W^{-(\gamma-1) / \gamma} \\
e^{\Psi} & =c_{1} S^{-2} W^{-1 / \gamma}
\end{aligned}
$$

where $c_{0}$ and $c_{1}$ are integration constants. The velocity function $V$ can be shown to be

$$
V=\frac{c_{1}}{c_{0}} z^{(2-\gamma) / \gamma} S^{-2} W^{(\gamma-2) / \gamma} .
$$


Equations (2.18)-2.23) reduce to ordinary differential equations for $S, S^{\prime}$ and $W$ :

$$
\begin{aligned}
\frac{V^{2}-(\gamma-1)}{\gamma} \frac{W^{\prime}}{W}=\frac{\gamma c_{1}^{2} W^{(\gamma-2) / \gamma}}{2 S^{4}}-\frac{2(\gamma-1)}{\gamma} & -2 V^{2} \frac{S^{\prime}}{S} \\
S^{\prime \prime}= & S^{\prime}\left(\frac{\gamma-2}{\gamma}-\frac{(\gamma-1) W^{\prime}}{\gamma W}\right) \\
& -\left(S+S^{\prime}\right)\left(\frac{2 S^{\prime}}{S}+\frac{W^{\prime}}{\gamma W}\right) \\
M= & W S^{2}\left(\gamma S^{\prime}+S\right), \\
M= & S\left[1+c_{0}^{-2} z^{2(2-\gamma) / \gamma} W^{2(\gamma-1) / \gamma}{S^{\prime}}^{2}\right. \\
& \left.-c_{1}^{-2} S^{4} W^{2 / \gamma}\left(S+S^{\prime}\right)^{2}\right]
\end{aligned}
$$

From Eqs. (2.29) and (2.30), we obtain the following relation between $S, S^{\prime}$ and $W$ :

$$
\begin{aligned}
W S^{2}\left(\gamma S^{\prime}+S\right) & =S\left[1+c_{0}^{-2} z^{2(2-\gamma) / \gamma} W^{2(\gamma-1) / \gamma}{S^{\prime}}^{2}\right. \\
& \left.-c_{1}^{-2} S^{4} W^{2 / \gamma}\left(S+S^{\prime}\right)^{2}\right]
\end{aligned}
$$

Using this and Eq. (2.29), we obtain another constraint between $M, S$ and $W$ :

$$
\begin{gathered}
\left(1-\frac{M}{W S^{3}}\right)^{2} V^{2}-\left(\gamma-1+\frac{M}{W S^{3}}\right)^{2} \\
+\gamma^{2} c_{1}^{2} W^{-2 / \gamma} S^{-6}\left(1-\frac{M}{S}\right)=0 .
\end{gathered}
$$

\section{EXACT SOLUTIONS}

\section{A. Friedmann solution}

The flat Friedmann solution in self-similar coordinates corresponds to

$$
S=S_{0} z^{-2 /(3 \gamma)}, \quad W=W_{0} z^{2},
$$

where $S_{0}$ and $W_{0}$ are constants. $V$ is determined by Eq. (2.26) as

$$
V=V_{0} z^{1-2 /(3 \gamma)}, \quad V_{0}=\frac{c_{1}}{c_{0}} S_{0}^{-2} W_{0}^{(\gamma-2) / \gamma} .
$$

and Eq. (2.28) holds trivially. From Eqs. (2.27) and (2.31), $S_{0}$ and $W_{0}$ must satisfy

$$
\begin{aligned}
\frac{2}{3 \gamma} V_{0}^{2} & =\frac{\gamma c_{1}^{2} W_{0}^{(\gamma-2) / \gamma}}{2 S_{0}^{4}} \\
1 & =c_{1}^{-2} S_{0}^{6} W_{0}^{2 / \gamma}\left(1-\frac{2}{3 \gamma}\right)^{2} .
\end{aligned}
$$

These equations are consistent provided $\gamma$ is not $2 / 3$ or 0 . Since $S_{0}$ and $W_{0}$ are determined in terms of $\gamma, c_{0}$ and $c_{1}$, where $c_{0}$ and $c_{1}$ are just gauge constants, there is no free parameter in this solution for a given value of $\gamma$.

Introducing constants $a_{0}$ and $b_{0}$ in place of $S_{0}$ and $W_{0}$, we obtain:

$$
\begin{aligned}
e^{\Phi} & =e^{\Phi_{\mathrm{F}}} \equiv a_{0}, \\
e^{\Psi} & =e^{\Psi_{\mathrm{F}}} \equiv b_{0} z^{-q}, \\
S & =S_{\mathrm{F}} \equiv \frac{b_{0}}{|1-q|} z^{-q}, \\
W & =W_{\mathrm{F}} \equiv \frac{4}{3 \gamma^{2} a_{0}^{2}} z^{2}, \\
M & =M_{\mathrm{F}} \equiv \frac{4 b_{0}^{3}}{9 \gamma^{2} a_{0}^{2}|1-q|^{3}} z^{2-3 q} .
\end{aligned}
$$

Here the constants $a_{0}$ and $b_{0}$ can be chosen arbitrarily and

$$
q \equiv \frac{2}{3 \gamma} .
$$

The constants $c_{0}$ and $c_{1}$ in Eqs. (2.24) and (2.25) are given in terms of $a_{0}$ and $b_{0}$ as

$$
\begin{aligned}
& c_{0}=a_{0}\left(\frac{4}{3 \gamma^{2} a_{0}^{2}}\right)^{(\gamma-1) / \gamma}, \\
& c_{1}=\frac{b_{0}^{3}}{(1-q)^{2}}\left(\frac{4}{3 \gamma^{2} a_{0}^{2}}\right)^{1 / \gamma},
\end{aligned}
$$

where Eqs. (3.1)-(3.4), (3.7) and (3.8) are used. One can put the metric into a more familiar form,

$$
d s^{2}=-d \tilde{t}^{2}+\tilde{t}^{2 q}\left(d \tilde{r}^{2}+\tilde{r}^{2} d \Omega^{2}\right),
$$

by introducing new coordinates

$$
\tilde{t}=a_{0} t, \quad \tilde{r}=a_{0}^{-q} b_{0} r^{1-q} /|1-q| .
$$

It should be noted that $\tilde{r}=\infty$ corresponds to $r=\infty$ for the decelerating case $(2 / 3<\gamma \leq 2)$ but to $r=0$ for the accelerating one $(0<\gamma<2 / 3)$. In the $\gamma=2 / 3$ case, the homothetic Killing vector is parallel to the fluid flow and these equations no longer apply [24].

There exists a finite non-zero $z_{1}$ where $V$ crosses 1 . Figure 1 shows the conformal diagram of the flat Friedmann solution for $0<\gamma<2 / 3$, including the similarity surfaces. We can see that the initial singularity is null. The similarity horizon $z=z_{1}$ corresponds to the cosmological event horizon, while $z=\infty$ and $r=\infty$ is a regular center. The dotted and dot-dashed lines denote the singularity and infinity, respectively.

\section{B. Kantowski-Sachs solution}

The Kantowski-Sachs solution in self-similar coordinates corresponds to

$$
S=S_{0} z^{-1}, \quad W=W_{0} z^{2} .
$$




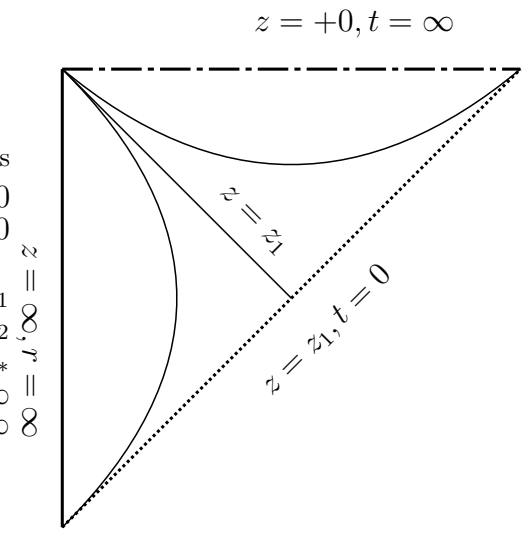

FIG. 1: The conformal diagram of the flat Friedmann solution for $0<\gamma<2 / 3$. There is a similarity horizon at $z=z_{1}$ $(>0)$, corresponding to the cosmological event horizon, and a regular center at $z=\infty$ and $r=\infty$. The initial singularity at $t=0$ is null, while null infinity is given by $t=\infty$. The dotted and dot-dashed lines denote the singularity and infinity, respectively. The thin solid curves and lines denote similarity surfaces, i.e. orbits of $z=$ constant.

Then Eq. (2.28) holds trivially and Eq. (2.31) yields

$$
(1-\gamma) W_{0} S_{0}^{3}=S_{0}\left[1+c_{0}^{-2} W_{0}^{2-2 / \gamma} S_{0}^{2}\right] .
$$

From Eq. (2.26), we have

$$
V=V_{0} z^{3-2 / \gamma}, \quad V_{0}=\frac{c_{1}}{c_{0}} S_{0}^{-2} W_{0}^{1-2 / \gamma},
$$

and hence $V \rightarrow 0$ as $z \rightarrow \infty$. Eq. (2.27) is trivially satisfied to lowest order but determines $W_{0}$ to the next lowest order:

$$
\left(\frac{1}{\gamma}-1\right) c_{0}^{-2}=\frac{\gamma}{4} W_{0}^{-1+2 / \gamma}
$$

Equations (3.16) and (3.18) then give

$$
\frac{(2-3 \gamma)(2-\gamma)}{4(1-\gamma)} W_{0} S_{0}^{2}=1,
$$

so we can obtain a physical solution only for $0<\gamma<2 / 3$ and there remains no free parameter in this case. This solution was first obtained in ref. 25], although that paper introduced different variables to deal with the unphysical solutions for the $2 / 3<\gamma<2$ case.

The Kantowski-Sachs solution in more standard coordinates can be written as [21]

$$
\begin{aligned}
d s^{2}= & -\frac{(2-3 \gamma)(2-\gamma)}{\gamma^{2}} d t^{2}+t^{4(1-\gamma) / \gamma} d \bar{r}^{2} \\
& +t^{2}\left(d \theta^{2}+\sin ^{2} \theta d \phi^{2}\right) \\
8 \pi G \mu & =\frac{4(1-\gamma)}{(2-3 \gamma)(2-\gamma) t^{2}} \\
2 G m & =\frac{4(1-\gamma)^{2} t}{(2-3 \gamma)(2-\gamma)}
\end{aligned}
$$

$$
V=\frac{|\gamma| z^{-(2-3 \gamma) / \gamma}}{\sqrt{(2-3 \gamma)(2-\gamma)}}
$$

where $\bar{r}$ is a radial coordinate. As expected, $V$ is only real for $0<\gamma<2 / 3$. In the Kantowski-Sachs solution, the area of the 2 -sphere with constant $t$ and $r$ does not depend on $r$ but only on $t$, so it expands with time. The topology of the constant $t$ spacelike hypersurface is $S^{2} \times$ $R$.

There again exists a non-zero finite $z_{1}$ where $V$ crosses 1. $r=\infty$ or $z=\infty$ for fixed $t(>0)$ can be analytically extended to negative $r$ or negative $z$ beyond $z= \pm \infty$. Figure 2 shows the conformal diagram of the (extended) Kantowski-Sachs solution for $0<\gamma<2 / 3$. The similarity surfaces are also shown. We can see that the initial singularity is null. There are two similarity horizons $z= \pm z_{1}$, corresponding to two cosmological event horizons. The corresponding conformal diagram (Fig. 15) in ref. [22] is incorrect.

$$
z=-0, t=\infty \quad z=+0, t=\infty
$$

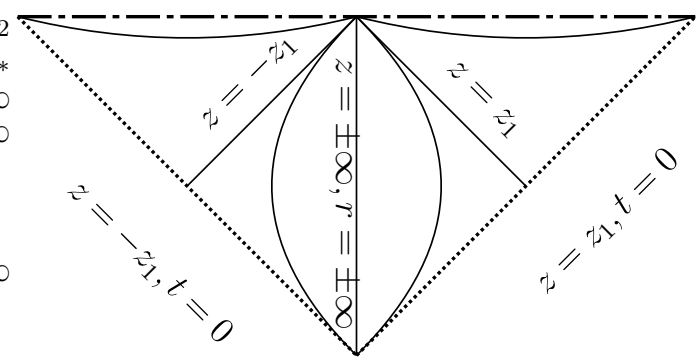

FIG. 2: The conformal diagram of the Kantowski-Sachs solution for $0<\gamma<2 / 3$. The solution is analytically extendible beyond $z= \pm \infty$ and $r= \pm \infty$. There are two similarity horizons $z= \pm z_{1}$, both corresponding to cosmological event horizons. The initial singularity at $t=0$ is null and future null infinity is given by $t=\infty$.

\section{Absence of static solution}

The static solution would need to have

$$
S=S_{0}, \quad W=W_{0},
$$

in which case Eq. (2.26) gives

$$
V=V_{0} z^{(2-\gamma) / \gamma}, \quad V_{0}=\frac{c_{1}}{c_{0}} S_{0}^{-2} W_{0}^{(\gamma-2) / \gamma} .
$$

Equations (2.27) and (2.31) then reduce to

$$
0=\frac{\gamma c_{1}^{2} W_{0}^{(\gamma-2) / \gamma}}{S_{0}^{4}}+2\left(\frac{1-\gamma}{\gamma}\right)
$$

and

$$
W_{0} S_{0}^{3}=S_{0}\left[1-c_{1}^{-2} S_{0} W_{0}^{2 / \gamma}\right]
$$


respectively. For $\gamma>1$, Eq. 3.25) can be satisfied and there is a static self-similar solution, but there is no such solution for $0 \leq \gamma<1$ However, as we will see later, the absence of an exact static solution does not preclude the possibility of an asymptotically static solution.

\section{ASYMPTOTIC BEHAVIORS}

\section{A. Friedmann asymptote for $z \rightarrow 0$}

We now focus on self-similar solutions which are asymptotic to the flat Friedmann model at large spatial distances, i.e. in which $\Phi, \Psi, S, W$ and $M$ approach the form given by Eqs. (3.5)-3.9) as $\tilde{r} \rightarrow \infty$. In this case, it is convenient to introduce new functions $A$ and $B$, defined by

$$
\begin{aligned}
W & =W_{\mathrm{F}}(z) e^{A(z)}, \\
S & =S_{\mathrm{F}}(z) e^{B(z)},
\end{aligned}
$$

which describe the deviations from the flat Friedmann solution. Equations (2.27), (2.28) and (2.26) can then be written as

$$
\begin{aligned}
A^{\prime}= & \frac{\gamma V^{2}\left[3 \gamma q^{2} e^{-(\gamma-2) A / \gamma}-2\left(q+2 B^{\prime}\right)\right]}{2\left[V^{2}-(\gamma-1)\right]}, \\
B^{\prime \prime}= & -\left(B^{\prime}-q\right)\left(1-q+B^{\prime}+\frac{\gamma-1}{\gamma} A^{\prime}\right) \\
& -\left(q+2 B^{\prime}+\frac{1}{\gamma} A^{\prime}\right)\left(1-q+B^{\prime}\right), \\
V= & \frac{b_{0}}{a_{0}} z^{1-q} e^{-2 B+(1-2 / \gamma) A},
\end{aligned}
$$

respectively. Equation (2.31) yields another relation:

$$
\begin{gathered}
\frac{4 b_{0}^{3}}{9 \gamma^{2} a_{0}^{2}|1-q|^{3}} z^{2-3 q} e^{A+3 B}\left(1+3 \gamma B^{\prime}\right) \\
=\frac{b_{0}}{|1-q|} z^{-q} e^{B}\left[1+\frac{b_{0}^{2}}{a_{0}^{2}(1-q)^{2}} z^{2-2 q}\right. \\
\quad \times e^{2(\gamma-1) A / \gamma+2 B}\left(-q+B^{\prime}\right)^{2} \\
\left.-\frac{1}{(1-q)^{2}} e^{2 A / \gamma+6 B}\left(1-q+B^{\prime}\right)^{2}\right] .
\end{gathered}
$$

The flat Friedmann solution is given by $A=B=0$ for all $z$. Spacelike infinity corresponds to $z \rightarrow \infty$ for the decelerating case $(2 / 3<\gamma \leq 2)$ and $z \rightarrow 0$ for the accelerating case $(0<\gamma<2 / 3)$. Note that $V \propto z^{1-2 /(3 \gamma)} \rightarrow \infty$ at large distances if $A$ and $B$ are finite. This means that $z=\infty(2 / 3<\gamma \leq 2)$ and $z=0(0<\gamma<2 / 3)$ are horizontal lines in a conformal diagram.

The asymptotic form of self-similar solutions which approach the flat Friedmann at large distances can be found by neglecting the $V^{-2}$ term and linearizing the equations with respect to $A, A^{\prime}, B^{\prime}$ and $B^{\prime \prime}$. (It is noted that $B$ does not need to be small.) From Eqs. (4.3) and (4.4), the linearized equations at spacelike infinity become

$$
\begin{aligned}
& 0=3 q^{2}(\gamma-2) A+4 B^{\prime}+\frac{2}{\gamma} A^{\prime}, \\
& 0=B^{\prime \prime}+3(1-q) B^{\prime}+\left(\frac{1-\gamma q}{\gamma}\right) A^{\prime}
\end{aligned}
$$

in both cases. These equations lead to two independent solutions and the general solution is given by a linear combination of these.

The first solution is

$$
\begin{aligned}
& A(z)=A_{1} z^{2(2-3 \gamma) /(3 \gamma)}+\cdots, \\
& B(z)=\beta+B_{1} z^{2(2-3 \gamma) /(3 \gamma)}+\cdots,
\end{aligned}
$$

where

$$
\begin{aligned}
& A_{1} \equiv(2-3 \gamma) B_{1}, \\
& B_{1} \equiv-\frac{a_{0}^{2}}{b_{0}{ }^{2}} \frac{\gamma(3 \gamma-2)}{4(3 \gamma+2)}\left(e^{-2 \beta}-e^{4 \beta}\right) .
\end{aligned}
$$

$\beta$ is a constant and the dots denote higher order terms. $A$ and $B$ converge at large distances in both the decelerating $(2 / 3<\gamma \leq 2)$ and accelerating $(0<\gamma<2 / 3)$ cases. This solution was first obtained in ref. [17] for the radiation case $(\gamma=4 / 3)$ and in ref. [19] for more general $\gamma$. However, it is not properly asymptotic to the flat Friedmann solution because Eq. (4.2) shows that there is a residual $e^{\beta}$ term in $S$ at infinity, so Eq. (2.12) implies that there is a solid angle deficit [20]. We describe solutions with this asymptotic behavior as asymptotically quasi-Friedmann.

The second solution is

$$
\begin{aligned}
& A \approx A_{0} z^{(2-\gamma) / \gamma}, \\
& B \approx-\frac{1}{6 \gamma} A_{0} z^{(2-\gamma) / \gamma},
\end{aligned}
$$

where $A_{0}$ is a constant and $x \approx y$ means $(x / y) \rightarrow 1$ in the relevant limit. The above solution was also found in ref. [26]. The decelerating solution must be discarded, since this diverges at large distances $(z \rightarrow \infty)$. On the other hand, the accelerating solution is "properly" asymptotic to Friedmann since $A$ and $B$ both converge to zero at large distances $(z \rightarrow 0)$, so there is no solid angle deficit. In the following, we generally omit the term "properly" and simply describe these solutions as asymptotically Friedmann.

We conclude that there is a 1-parameter family of asymptotically Friedmann solutions at large distances for $0<\gamma<2 / 3$. Note that, in the positive pressure case $(\gamma>1)$, it is well known that there is no physical solution which is exactly Friedmann at sufficiently large but finite distances [17]. Although one might envisage attaching an interior black hole solution to an exterior Friedmann solution at a sonic point (since there can be a discontinuity there), no such solution is possible. This conclusion trivially applies when the pressure is negative $(\gamma<1)$ because there are no sound-waves in this case. 


\section{B. Friedmann asymptote for $z \rightarrow \infty$}

We are also interested in solutions which are asymptotically Friedmann at small distances from the origin. We therefore seek solutions which have

$$
S \approx S_{0} z^{-2 /(3 \gamma)}, \quad W \approx W_{0} z^{2}
$$

for $z \rightarrow \infty$ and $0<\gamma<2 / 3$, where the constants $S_{0}$ and $W_{0}$ may be different from the exact flat Friedmann case. Equation (2.26) implies

$$
V \approx V_{0} z^{1-2 /(3 \gamma)}, \quad V_{0}=\frac{c_{1}}{c_{0}} S_{0}^{-2} W_{0}^{(\gamma-2) / \gamma} .
$$

The exact Friedmann relation 3.4 still applies but Eq. 2.27) now yields to lowest order

$\frac{2}{3 \gamma} V_{0}^{2}+\frac{1-\gamma}{\gamma}\left(\frac{W^{\prime}}{W}-2\right) z^{-2(1-2 /(3 \gamma))}=\frac{\gamma c_{1}^{2} W_{0}^{(\gamma-2) / \gamma}}{2 S_{0}^{4}}$

Hence $\left(W^{\prime} / W\right)-2$ is either proportional to $z^{2-4 /(3 \gamma)}$ or falls off even faster. Therefore Eq. 2.28) implies the following asymptotic behavior:

$$
\begin{aligned}
S & =S_{0} z^{-2 /(3 \gamma)}\left[1+S_{1} z^{2(1-2 /(3 \gamma))}+\cdots\right] \\
W & =W_{0} z^{2}\left[1+W_{1} z^{2(1-2 /(3 \gamma))}+\cdots\right]
\end{aligned}
$$

where

$$
\begin{aligned}
& \frac{2}{3 \gamma} V_{0}^{2}+\frac{1-\gamma}{\gamma} W_{1}=\frac{\gamma c_{1}^{2} W_{0}^{(\gamma-2) / \gamma}}{2 S_{0}^{4}} \\
& S_{1}=-\frac{1}{5 \gamma(3-2 \gamma)} W_{1} .
\end{aligned}
$$

All coefficients of higher order terms are determined from $S_{0}$ and $W_{0}$. Since the value for $S_{0}$ can be different from the exact Friedmann case, there is a 1-parameter family of asymptotically quasi-Friedmann solutions at small distances. Since $V \rightarrow 0$ as $z \rightarrow \infty, z=\infty$ is a vertical line in a conformal diagram. These solutions can be interpreted as self-similar models with a regular center as $r \rightarrow 0$ for fixed $t \neq 0$. On the other hand, in the limit $t \rightarrow 0$ for fixed $r \neq 0$, they correspond to simultaneous big bang models in the comoving time-slicing. If $S_{0}$ had the exact Friedmann value, we would have Friedmann itself, so this is the only solution which is properly asymptotically Friedmann.

\section{Static asymptote for $z \rightarrow \infty$}

As we have seen, there exists no static self-similar solution for $0<\gamma<2 / 3$. However, we will show that there are still solutions which can be described as asymptotically quasi-static. In this case, we assume

$$
W \approx W_{0}>0, \quad S \approx S_{0}>0
$$

as $z \rightarrow \infty$. Only if $S_{0}$ and $W_{0}$ are the same as in the static solution do we describe such solutions as asymptotically static. Equation (2.26) implies

$$
V \approx V_{0} z^{(2-\gamma) / \gamma}, \quad V_{0}=\frac{c_{1}}{c_{0}} z^{(2-\gamma) / \gamma} S_{0}^{-2} W_{0}^{(\gamma-2) / \gamma} .
$$

This solution can be compatible with Eq. (2.27) if and only if

$$
\frac{1}{\gamma} \frac{W^{\prime}}{W}+2 \frac{S^{\prime}}{S} \propto z^{-2(2-\gamma) / \gamma}
$$

as $z \rightarrow \infty$. This combination appears in the second term on the right-hand side of Eq. (2.28) and this can be regarded as a higher order term. It then follows that

$$
S=S_{0}+S_{1} z^{1-2 / \gamma}+S_{2} z^{2(1-2 / \gamma)}+\cdots
$$

From Eq. (4.23), $W$ must be of the form

$$
W=W_{0}+W_{1} z^{1-2 / \gamma}+W_{2} z^{2(1-2 / \gamma)}+\cdots,
$$

where

$$
\frac{1}{\gamma} \frac{W_{1}}{W_{0}}+2 \frac{S_{1}}{S_{0}}=0
$$

From Eq. (2.31), we obtain the relation

$W_{0} S_{0}^{3}=S_{0}\left[1+\left(1-\frac{2}{\gamma}\right)^{2} c_{0}^{-2} W_{0}^{2(1-2 / \gamma)} S_{1}^{2}-c_{1}^{-2} S_{0}^{6} W_{0}^{2 / \gamma}\right]$.

To lowest order Eq. (2.27) becomes

$$
2 V_{0}^{2}\left(1-\frac{2}{\gamma}\right)\left[\frac{1}{\gamma} \frac{W_{2}}{W_{0}}+2 \frac{S_{2}}{S_{0}}\right]=\frac{\gamma c_{1}^{2} W_{0}^{1-2 / \gamma}}{2 S_{0}^{4}}+\frac{2(1-\gamma)}{\gamma}
$$

and to the next lowest order Eq. (2.28) becomes

$$
\begin{aligned}
2\left(1-\frac{2}{\gamma}\right)^{2} S_{2} & =-\left(1-\frac{1}{\gamma}\right)\left(1-\frac{2}{\gamma}\right)^{2} S_{1} W_{1} \\
& \left.-2\left(1-\frac{1}{\gamma}\right) S_{0}\left(\frac{2 S_{2}}{S_{0}}+\frac{W_{2}}{\gamma W_{0}}\right) 4.29\right)
\end{aligned}
$$

$S_{1}$ and $W_{1}$ are determined from Eqs. (4.26) and (4.27) in terms of $S_{0}$ and $W_{0}$, while $S_{2}$ and $W_{2}$ are determined by Eqs. (4.28) and 4.29) in terms of $S_{0}, S_{1}, W_{0}$ and $W_{1}$. All higher order terms are determined in terms of $S_{0}$ and $W_{0}$. Hence there is a 2-parameter family of asymptotically quasi-static solutions. Since there is no exact static solution, there are no asymptotically static ones. Also $V \rightarrow \infty$ as $z \rightarrow \infty$ for $0<\gamma<2 / 3$, so $z=\infty$ is a horizontal line in a conformal diagram.

If we consider the analytic continuation beyond $z=\infty$ into the negative $z$ region, the metric should remain analytic in terms of the local inertial Cartesian coordinates. For asymptotically quasi-static solutions, one should consider the analytic continuation beyond $t=+0$ because 
$S=R / r$ and $W=8 \pi G \mu r^{2}$ are finite. For this purpose, it is convenient to see how the proper time $\tau$ behaves for fixed $r>0$. For this class of solutions, Eq. (2.24) implies

$$
e^{\Phi}=c_{0} z^{-2(1-\gamma) / \gamma} W_{0}^{(1-\gamma) / \gamma},
$$

so $\tau \propto t^{-1+2 / \gamma}$. When we continue the solution analytically to the negative $t$ region beyond $t= \pm 0$, this means that $\tau \approx \pm C_{r}|t|^{-1+2 / \gamma}$, where $C_{r}$ is a positive constant depending on $r$ and the upper (lower) sign corresponds to the positive (negative) $t$. To express $W$ and $S$ as analytic functions of $\tau$, we use the following unique continuation:

$$
\begin{aligned}
S & =S_{0} \pm S_{1}|z|^{1-2 / \gamma}+S_{2}|z|^{2(1-2 / \gamma)}+\cdots \\
W & =W_{0} \pm W_{1}|z|^{1-2 / \gamma}+W_{2}|z|^{2(1-2 / \gamma)}+\cdots
\end{aligned}
$$

This expression gives a Taylor series expansion around $\tau=0$ in terms of $\tau$. Because of the presence of the odd powers of $\tau$, this is not reflection-symmetric about $t=0$.

On the other hand, in the limit $r \rightarrow \infty$ for fixed $t \neq 0$, one has a vacuum of infinite radius which is not asymptotically flat because $m / R$ approaches a non-zero constant. We call this quasi-static spacelike infinity. In Paper II, we will see that there are a class of solutions describing a Friedmann universe emergent from a white hole, where a Friedmann spacelike infinity and a quasistatic spacelike infinity are connected.

\section{Kantowski-Sachs asymptote for $z \rightarrow \infty$}

For the asymptotically quasi-Kantowski-Sachs solutions, we assume

$$
S \approx S_{0} z^{-1}, \quad W \approx W_{0} z^{2}
$$

as $z \rightarrow \infty$. From Eq. (2.26), we have

$$
V \approx V_{0} z^{3-2 / \gamma}, \quad V_{0}=\frac{c_{1}}{c_{0}} S_{0}^{-2} W_{0}^{1-2 / \gamma} z^{3-2 / \gamma},
$$

and hence $V \rightarrow 0$ as $z \rightarrow \infty$. Equation (2.27) is trivially satisfied to lowest order but to the next order requires either

$$
\frac{W^{\prime}}{W}-2 \propto z^{2(3-2 / \gamma)}
$$

or that the right-hand side falls off even faster than this. Equations (2.28) and (4.35) imply

$$
\begin{aligned}
S & =S_{0} z^{-1}\left(1+S_{1} z^{3-2 / \gamma}+S_{2} z^{2(3-2 / \gamma)}+\cdots\right. \\
W & =W_{0} z^{2}\left(1+W_{2} z^{2(3-2 / \gamma)}+\cdots\right)
\end{aligned}
$$

Equation (2.31) yields

$$
\begin{aligned}
(1-\gamma) W_{0} S_{0}^{3}= & S_{0}\left[1+c_{0}^{-2} W_{0}^{2(\gamma-1) / \gamma} S_{0}^{2}\right. \\
& \left.-\left(3-\frac{2}{\gamma}\right)^{2} c_{1}^{-2} S_{0}^{6} W_{0}^{2 / \gamma} S_{1}^{2}\right]
\end{aligned}
$$

while Eq. (2.27) gives

$$
\frac{2(1-\gamma)}{\gamma} V_{0}^{2}+2 \frac{1-\gamma}{\gamma}\left(3-\frac{2}{\gamma}\right) W_{2}=\frac{\gamma c_{1}^{2} W_{0}^{1-2 / \gamma}}{2 S_{0}^{4}} .
$$

At second order Eq. (2.28) becomes

$$
\left(4-\frac{3}{\gamma}\right) S_{2}=-\left(1-\frac{1}{\gamma}\right)\left(3-\frac{2}{\gamma}\right)\left(W_{2}+S_{1}^{2}\right) .
$$

Hence the coefficients of all higher order terms are determined in terms of $S_{0}$ and $W_{0}$. This means there is a 2-parameter family of asymptotically quasi-KantowskiSachs solutions. If the values for $S_{0}$ and $W_{0}$ are the same as those for the exact Kantowski-Sachs solution, all coefficients of higher order terms vanish, so the solution is also exactly Kantowski-Sachs. In other words, the only solution which is asymptotically Kantowski-Sachs is Kantowski-Sachs itself. On the other hand, even if the first order terms vanish, i.e. $S_{1}=0$, we can see that $W_{2}$ may not vanish, so $S_{0}$ and $W_{0}$ are different from their Kantowski-Sachs values. Since $V \rightarrow 0$ as $z \rightarrow \infty$ for $0<\gamma<2 / 3, z=\infty$ is a vertical line in a conformal diagram.

For asymptotically quasi-Kantowski-Sachs solutions, one can consider the analytic continuation beyond $r=\infty$ because $z S=R / t$ and $W / z^{2}=8 \pi G \mu t^{2}$ are non-zero and finite for fixed $t \neq 0$. It is useful to see how the proper length $\lambda$ changes around $r=\infty$ for fixed $t \neq 0$. Equation (2.25) implies

$$
e^{\Psi}=c_{1} z^{2-2 / \gamma} S_{0}^{-2} W_{0}^{-1 / \gamma},
$$

so $\lambda \propto r^{3-2 / \gamma}$. This means that, when we continue the solution analytically to the negative $r$ region beyond $r=\infty$, one has $\lambda \approx \mp C_{t}|r|^{3-2 / \gamma}$, where $C_{t}$ is a positive constant depending on $t$ and the upper (lower) sign corresponds to positive (negative) $r$. To obtain analytic expressions for $W$ and $S$ in terms of $\lambda$, we use the following continuation:

$$
\begin{aligned}
S & =S_{0} z^{-1}\left(1 \pm S_{1}|z|^{3-2 / \gamma}+S_{2}|z|^{2(3-2 / \gamma)}+\cdots\right. \\
W & =W_{0} z^{2}\left(1+W_{2}|z|^{2(3-2 / \gamma)}+\cdots\right)
\end{aligned}
$$

The above expression gives a Taylor series expansion around $\lambda=0$. Since $W_{1}=0$ in the above expression, $W / z^{2}=8 \pi G t^{2}$ generically has an extremum at $z= \pm \infty$, while $z S=R / t$ does not. So this expression is not reflection-symmetric with respect to $r= \pm \infty$. On the other hand, in the limit $t \rightarrow 0$ for fixed $r \neq 0$, we have $R \rightarrow 0$ and $\mu \rightarrow \infty$, which corresponds to an initial singularity. In Paper II, we will see that this analytical continuation is crucial for obtaining black hole solutions embedded in a Friedmann background as well as a class of wormhole solutions connecting one Friedmann spacelike infinity to another quasi-Friedmann spacelike infinity. 


\section{E. Constant-velocity asymptote for $z \rightarrow \infty$}

Here we seek solutions in which $V$ tends to a finite positive value $V_{\infty}$ as $z \rightarrow \infty$. Differentiating Eq. (2.26) and noting that $V^{\prime} / V \rightarrow 0$ as $z \rightarrow \infty$, we have

$$
\frac{W^{\prime}}{W} \approx 1-\left(\frac{2 \gamma}{2-\gamma}\right) \frac{S^{\prime}}{S}
$$

Substituting this relation into Eq. (2.28), we find to lowest order

$$
\frac{S^{\prime \prime}}{S} \approx-\frac{1}{\gamma}-\frac{4(1-\gamma)}{(2-\gamma)}\left(\frac{S^{\prime}}{S}\right)^{2}-\frac{2\left(2-\gamma^{2}\right)}{\gamma(2-\gamma)} \frac{S^{\prime}}{S}
$$

To lowest order this ordinary differential equation has a general solution of the form

$$
S \approx S_{0}^{+} z^{p_{+}}+S_{0}^{-} z^{p_{-}}
$$

where $S_{0, \pm}$ are arbitrary constants and

$$
p_{ \pm}=\frac{-\left(2-\gamma^{2}\right) \pm \sqrt{(1-\gamma)\left(4-8 \gamma+4 \gamma^{2}-\gamma^{3}\right)}}{(6-5 \gamma) \gamma} .
$$

One can show that the square root is real for $0<\gamma<$ 2/3. Also Eq. (2.31) is consistent only if the first term dominates the second term in Eq. (4.46), so $S_{0}^{+}=0$ is excluded.

One can easily show that the first term on the righthand side of Eq. (2.27) converges to zero for $0<\gamma<2 / 3$ and so

$$
V_{\infty}=\frac{\gamma(1-\gamma)+\sqrt{(1-\gamma)\left(4-8 \gamma+4 \gamma^{2}-\gamma^{3}\right)}}{2-\gamma} .
$$

This is plotted in Fig. 3 Note that the solution with similar asymptotic behavior for $\gamma>6 / 5$ [4] has the other branch of the square root. For this solution, we have

$$
S \approx S_{0} z^{p_{+}}, \quad W \approx W_{0} z^{1-2 \gamma p_{+} /(2-\gamma)} .
$$

The condition $V \rightarrow V_{\infty}$ yields a relation between $S_{0}$ and $W_{0}$ :

$$
V_{\infty}=\frac{c_{1}}{c_{0}} S_{0}^{-2} W_{0}^{(\gamma-2) / \gamma} .
$$

There is no other relation between them, so there is a 1-parameter family of solutions with this asymptotic behavior. Since $V \rightarrow V_{\infty}<1$ as $z \rightarrow \infty$ for $0<\gamma<2 / 3$, $z=\infty$ is a vertical line in a conformal diagram.

We now discuss the physical significance of this asymptotic solution. As $\gamma$ increases from 0 to $2 / 3, p_{+}$decreases from $-1 / 2$ to $-3 / 4$, while $V_{\infty}$ decreases from 1 to $1 / 3$. Because $M \propto z^{1+(6-5 \gamma) p_{+} /(2-\gamma)}$, we have $R=r S \rightarrow \infty$, $\mu=W /\left(8 \pi G r^{2}\right) \rightarrow 0, m=r M /(2 G) \rightarrow \infty$ and $2 m / R=M / S \rightarrow \infty$ as $r \rightarrow \infty$ for fixed $t \neq 0$. Therefore, this solution approaches a vacuum region with infinite physical radius. However, because the fall-off of

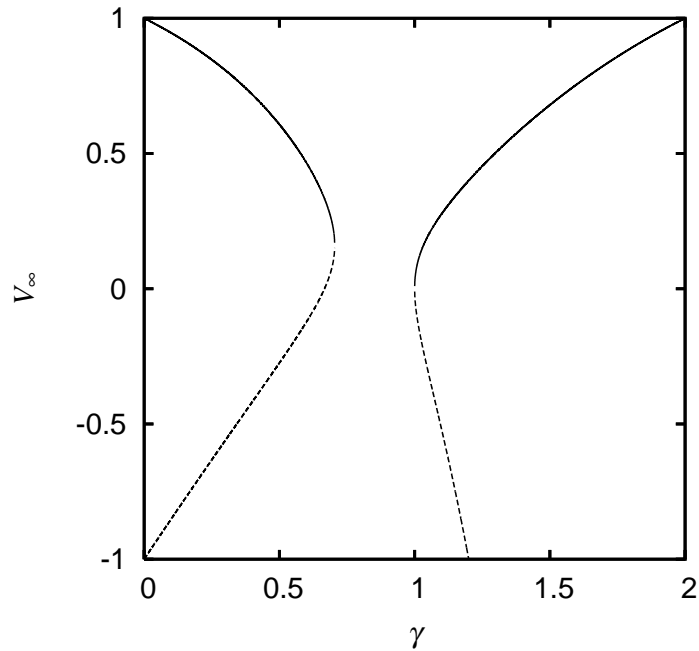

FIG. 3: $V_{\infty}$ for the asymptotically constant-velocity solution as a function of $\gamma$. Although only the part of the solid curve with $0<\gamma<2 / 3$ is relevant to the present analysis, it is extended to $0<\gamma<2$ for completeness. The other root for $V_{\infty}$ is also plotted with a dashed line but this may be unphysical.

the energy density $\mu$ is very slow, the solution is far from asymptotically flat. In fact, both $m$ and $m / R$ diverge to infinity. This solution cannot be analytically extended beyond $z=\infty$ because $R$ diverges to infinity as $r \rightarrow \infty$ for fixed $t \neq 0$, while $\mu$ diverges to infinity as $t \rightarrow 0$ for fixed $r \neq 0$. We describe these as asymptotically constant-velocity solutions. The infinity reached as $r \rightarrow \infty$ for fixed $t \neq 0$ will be called the constant-velocity spacelike infinity (cf. the asymptotically Minkowski solutions for $\gamma>6 / 5$ described in CC).

\section{F. Singular asymptote for $z \rightarrow z_{*}$}

We assume that $\ln |S|$ diverges as $z \rightarrow z_{*}$, while $V$ tends to a finite value $V_{*}$. Then Eq. (2.26) implies

$$
\frac{W^{\prime}}{W} \approx-\frac{2 \gamma}{2-\gamma} \frac{S^{\prime}}{S}
$$

and Eq. (2.28) yields to lowest order

$$
\begin{aligned}
S & \approx S_{0}|Z|^{(2-\gamma) /(6-5 \gamma)}, \\
W & \approx W_{0}|Z|^{-2 \gamma /(6-5 \gamma)},
\end{aligned}
$$

where

$$
Z \equiv \ln \frac{z}{z_{*}} .
$$

Equation (2.27) implies $V_{*}^{2}=1$, while Eq. (2.29) gives

$$
M \rightarrow M_{0}= \pm \frac{\gamma(2-\gamma)}{6-5 \gamma} W_{0} S_{0}^{3}
$$


where the upper (lower) sign corresponds to positive (negative) $Z$. From Eq. (2.26), we have

$$
\frac{c_{1}^{2}}{c_{0}^{2}}\left|z_{*}\right|^{2(2-\gamma) / \gamma} S_{0}^{-4} W_{0}^{2(\gamma-2) / \gamma}=1
$$

and hence $\left|z_{*}\right|$ is determined from $S_{0}$ and $W_{0}$. To next lowest order, Eq. (2.28) implies

$$
\begin{gathered}
S=S_{0}|Z|^{(2-\gamma) /(6-5 \gamma)}\left[1+S_{1}|Z|^{(2-3 \gamma) /(6-5 \gamma)}+\cdots(4,57)\right. \\
W=W_{0}|Z|^{-2 \gamma /(6-5 \gamma)}\left[1+W_{1}|Z|^{(2-3 \gamma) /(6-5 \gamma)}+\cdots(-4.58)\right.
\end{gathered}
$$

where

$$
S_{1}=-\frac{(2-\gamma)}{2(4-3 \gamma)} W_{1}
$$

Through Eq. (2.26), we obtain

$$
V=1+V_{1}|Z|^{(2-3 \gamma) /(6-5 \gamma)}+\cdots,
$$

where

$$
V_{1}=-2 S_{1}-\left(\frac{2-\gamma}{\gamma}\right) W_{1}
$$

and hence

$$
\begin{aligned}
S_{1} & =\frac{\gamma}{8(1-\gamma)} V_{1}, \\
W_{1} & =-\frac{\gamma(4-3 \gamma)}{4(2-\gamma)(1-\gamma)} V_{1} .
\end{aligned}
$$

To next lowest order Eq. 2.27) then gives

$$
V_{1}= \pm\left(\frac{6-5 \gamma}{2-\gamma}\right) \frac{\gamma c_{1}^{2} W_{0}^{-(2-\gamma) / \gamma}}{2 S_{0}^{4}}
$$

which is consistent with Eq. (2.31).

We describe these solutions as asymptotically singular. For fixed $r \neq 0$, we have $R=r S \rightarrow 0, \mu=W /\left(8 \pi G r^{2}\right) \rightarrow$ $\infty$ and $m=r M /(2 G) \approx r M_{0} /(2 G)$ as $Z \rightarrow 0$. From Eq. (4.55), we have

$$
m \approx \frac{r M_{0}}{2 G}= \pm \frac{\gamma(2-\gamma)}{6-5 \gamma} W_{0} S_{0}^{2} R
$$

and so the mass is positive (negative) for $Z \rightarrow 0^{+}$ $\left(Z \rightarrow 0^{-}\right)$. (Note that $S$ can be negative but $R=r S$ is positive even after the extension to the negative $r$ region.) Since the physical properties of these two limits are very different, we distinguish these two cases as asymptotically positive-mass singular and asymptotically negative-mass singular. For the positive-mass singular case, $Z \rightarrow 0^{+}, V \rightarrow 1^{+}$and $m>0$; for the negative case, $Z \rightarrow 0^{-}, V \rightarrow 1^{-}$and $m<0$. Although $V \rightarrow 1$ in the limit $Z \rightarrow 0$, the first component of the metric (2.13) is

$$
\begin{aligned}
& -\left(1-V^{2}\right) e^{2 \Phi} \\
\approx & 2 c_{0}^{2}\left|z_{*}\right|^{4(\gamma-1) / \gamma} W_{0}^{2(1-\gamma) / \gamma} V_{1}|Z|^{-(2-\gamma) /(6-5 \gamma(4) .66)}
\end{aligned}
$$

which tends to $\pm \infty$. Hence the similarity surface becomes spacelike (timelike) for the positive (negative) mass case. We will see in Paper II that the positivemass singular behavior is associated with black hole and white hole singularities, while the negative-mass one is associated with naked singularities.

It should be noted, however, that the mass of the singularity is not constant but is proportional to the time coordinate $t$. There is a 2-parameter family of solutions belonging to this class.

\section{SUMMARY AND DISCUSSION}

In this paper, we have analyzed spherically symmetric self-similar spacetimes. We have shown that the metric of these spacetimes is conformally static, with the conformal structure being determined by the form of the velocity function $V$. The homothetic Killing vector here plays an analogous role to the Killing vector in the static case. We have expressed the Einstein field equations in the comoving approach as ordinary differential equations and analytically investigated the possible asymptotic behavior of perfect fluid solutions with $p=(\gamma-1) \mu$ and $0<\gamma<2 / 3$. In Paper II, we focus on solutions which are asymptotic to the flat Friedmann universe at large distances because we are interested in solutions embedded in an accelerating Friedmann background [13].

These solutions can be understood in terms of the "complete classification" provided by Carr and Coley [4] . However, this classification mainly applies for perfect fluids with positive pressure and we have extended it to $0<\gamma<2 / 3$. We find there are eight possible asymptotic behaviors at small, intermediate and large distances in this class of solutions. However, it should be stressed that there are other asymptotic behaviors, such as KantowskiSachs asymptotes at $z \rightarrow \pm 0$, and these are discussed elsewhere [27].

Tables [1 and II summarize the results. Table I lists two exact solutions: Friedmann (F) and KantowskiSachs (KS). Table II lists eight asymptotic behaviors: asymptotically Friedmann (F) and asymptotically quasi-Friedmann (QF) for $z \rightarrow 0$, asymptotically quasi-Friedmann (QF), asymptotically quasi-static (QS), asymptotically quasi-Kantowski-Sachs (QKS) and asymptotically constant-velocity $(\mathrm{CV})$ for $z \rightarrow \infty$, asymptotically positive-mass singular (PMS) and asymptotically negative-mass singular (NMS) for $z \rightarrow z_{*}$.

There are three key differences from the positive pressure case. First, there is no sonic point in the negativepressure case and hence no additional ambiguity associated with the sonic point. Second, these solutions can be properly asymptotic to the flat Friedmann model, in the sense that there is no solid angle deficit. This contrasts with the positive pressure case, where the solutions are only asymptotically quasi-Friedmann. Third, the existence of exact and asymptotically Kantowski-Sachs solutions, which can be extended beyond the timelike hy- 
TABLE I: Self-similar exact solutions for $0<\gamma<2 / 3$. The form of $S, W$ and $V$, the number of parameters, the analytic continuation and the causal structure are shown. There is no arbitrary parameter in these solutions.

\begin{tabular}{|c||c|c|c|c|c|}
\hline Name & $S$ & $W$ & $V$ & Continuation & Structure \\
\hline \hline $\mathrm{F}$ & $z^{-\frac{2}{3 \gamma}}$ & $z^{2}$ & $z^{1-\frac{2}{3 \gamma}}$ & None & Fig. 1 \\
\hline $\mathrm{KS}$ & $z^{-1}$ & $z^{2}$ & $z^{3-\frac{2}{\gamma}}$ & $r= \pm \infty$ & Fig. 2 \\
\hline
\end{tabular}

persurface $z= \pm \infty$ along the spacelike direction, leads to physically interesting solutions embedded in a Friedmann background. The analytical results of this paper play a crucial role in Paper II, where we investigate the new solutions numerically and interpret them physically.

Finally, it should be emphasized that the matter model considered here suffers from dynamical instability for small-scale perturbation. For a perfect fluid with equation of state $p=(\gamma-1) \mu$, the derivative $d p / d \mu=\gamma-1$ is negative for $\gamma<1$, which violates the condition for dynamically stable equilibrium. We can also discuss this instability in terms of linear perturbation analysis. Consider a density perturbation with the dependence $e^{i(\omega t-\boldsymbol{k} \cdot \boldsymbol{x})}$ to linear order in flat spacetime. Then the dispersion relation $\omega^{2}=(\gamma-1) \boldsymbol{k}^{2}$ is obtained. Since the wave-number $\boldsymbol{k}$ is real, $\omega= \pm i \sqrt{1-\gamma}|\boldsymbol{k}|$ for $\gamma<1$, the lower sign corresponding to exponential growth of the perturbation. Since this instability exists for arbitrarily short wavelengths, the system is unstable even in the presence of gravity, except for the case of a cosmological constant $(\gamma=0)$. This means that the matter fields discussed in this paper can only be valid (i.e. dynamically stable) at larger scales. In fact, there are important physical models which have an effectively negative pressure through coarse-graining but which are still absolutely stable at small scales. For example, the effective equations of state for domain wall networks and string networks correspond to $\gamma=1 / 3$ and $\gamma=2 / 3$, respectively [28].

It should also be noted that this small-scale dynamical instability does not appear for a quintessence field (i.e. a scalar field with a flat potential). For example, suppose the scalar potential is given by $V=V_{0} \exp (\sqrt{8 \pi} \lambda \phi)$ $(\lambda>0)$. This can accelerate the Friedmann universe for $\lambda^{2}<2$. The dispersion relation for the scalar field perturbation in flat spacetime becomes $\omega^{2}=\boldsymbol{k}^{2}+8 \pi \lambda^{2} V\left(\phi_{0}\right)$, where $\phi_{0}(t, \boldsymbol{x})$ denotes the background scalar field and the short wavelength approximation is adopted. Thus, this scalar field is stable against short wavelength perturbations.

\section{Acknowledgments}

The authors would like to thank P. Ivanov, H. Kodama, H. Koyama, M. Siino and T. Tanaka for useful comments. HM and TH are supported by the Grant-in-Aid for Sci- entific Research Fund of the Ministry of Education, Culture, Sports, Science and Technology, Japan (Young Scientists (B) 18740162 (HM) and 18740144 (TH)). HM was also supported by the Grant No. 1071125 from FONDECYT (Chile). CECS is funded in part by an institutional grant from Millennium Science Initiative, Chile, and the generous support to CECS from Empresas CMPC is gratefully acknowledged. BJC thanks the Research Center for the Early Universe at the University of Tokyo for hospitality received during this work.

\section{APPENDIX A: COMPARISON WITH CARR-COLEY ANALYSIS.}

Carr and Coley 7] have also attempted to classify perfect-fluid spherically symmetric self-similar solutions with $0<\gamma<1$, although they have not investigated the solutions numerically (as we do in Paper II). Their analysis essentially extends the CC classification [4] of solutions with $1<\gamma<2$ and, in principle, includes the $0<\gamma<2 / 3$ case discussed here. However, a comparison with our results requires care because CC use a different system of notation from us. This appendix gives the relationship between the two systems and points out that the analysis in ref. [7] is not complete.

$\mathrm{CC}$ use the same comoving coordinates $(r, t, \theta, \phi)$ as us and the same similarity variable $z=r / t$, although a dot rather than a prime denotes $d / d \ln z$. They also use the same symbols for the velocity function $V$, pressure $p$, density $\mu$ and areal radial distance $R=r S$. Otherwise the mapping from our variables to theirs is as follows:

$$
\begin{array}{r}
\Phi \rightarrow \nu, \quad \Psi \rightarrow \lambda, \quad M \rightarrow M S \\
P \rightarrow 8 \pi G P / S^{2}, \quad W \rightarrow 8 \pi G W / S^{2} .
\end{array}
$$

They also express the field equations as ODEs in the quantities $\left(S, S^{\prime}, W\right)$ but they introduce the variable

$$
x \equiv\left(4 \pi \mu r^{2}\right)^{-(1+\gamma) /(2+\gamma)}
$$

instead of $W$. Note that the ODEs are expressed in terms of $(S, M, W)$ in ref. [11].

CC correctly identify Friedmann and Kantowski-Sachs as the only exact power-law self-similar solutions and they look for more general solutions which are asymptotic to these. They also point out that there is no exact static solutions for $0<\gamma<1$ but they do not notice that there are still asymptotically quasi-static solutions and this is the main reason why their classification is incomplete. Although CC appreciate the importance of extending some solutions from $z>0$ to $z<0$, they always take the variable $r$ to be positive, whereas we allow it to be negative.

Their analysis of the asymptotic solutions at large and small distances is closely related to ours and explicitly includes the asymptotically Friedmann and asymptotically Kantowski-Sachs solutions for $0<\gamma<2 / 3$. However, they do not distinguish between the asymptoti- 
TABLE II: The possible asymptotic behaviors of asymptotically Friedmann self-similar solutions for $0<\gamma<2 / 3$. The limiting value of $z$ and the form of $S, W$ and $V$, the number of parameters, the analytic continuation, the causal structure and the physical distance are shown. For the physical distance, the limiting values of $z S=R / t$ are shown. The values of $p_{+}=p_{+}(\gamma)$ and $V_{\infty}=V_{\infty}(\gamma)$ are given in the text.

\begin{tabular}{|c||c|c|c|c|c|c|c|c|}
\hline Name & $\mathrm{z}$ & $S$ & $W$ & $V$ & \#param & Extension & Structure & Distance \\
\hline \hline $\mathrm{F}$ & \pm 0 & $z^{-\frac{2}{3 \gamma}}$ & $z^{2}$ & $z^{1-\frac{2}{3 \gamma}}$ & 1 & None & Spacelike & $\infty$ \\
\hline $\mathrm{QF}$ & \pm 0 & $z^{-\frac{2}{3 \gamma}}$ & $z^{2}$ & $z^{1-\frac{2}{3 \gamma}}$ & 1 & None & Spacelike & $\infty$ \\
\hline $\mathrm{QF}$ & $\pm \infty$ & $z^{-\frac{2}{3 \gamma}}$ & $z^{2}$ & $z^{1-\frac{2}{3 \gamma}}$ & 1 & None & Timelike & 0 \\
\hline $\mathrm{QS}$ & $\pm \infty$ & $\mathrm{const}$ & $\mathrm{const}$ & $z^{\frac{2-\gamma}{\gamma}}$ & 2 & $t= \pm 0$ & Spacelike & $\infty$ \\
\hline $\mathrm{QKS}$ & $\pm \infty$ & $z^{-1}$ & $z^{2}$ & $z^{3-\frac{2}{\gamma}}$ & 2 & $r= \pm \infty$ & Timelike & Intermediate \\
\hline $\mathrm{CV}$ & $\pm \infty$ & $z^{p_{+}}$ & $z^{1-\frac{2 \gamma p}{2-\gamma}}$ & $V_{\infty}$ & 1 & None & Timelike & $\infty$ \\
\hline $\mathrm{PMS}$ & $z_{*}$ & $\left|z-z_{*}\right|^{\frac{2-\gamma}{6-5 \gamma}}$ & $\left|z-z_{*}\right|^{-\frac{2 \gamma}{6-5 \gamma}}$ & 1 & 2 & None & Spacelike & 0 \\
\hline $\mathrm{NMS}$ & $z_{*}$ & $\left|z-z_{*}\right|^{\frac{2-\gamma}{6-5 \gamma}}$ & $\left|z-z_{*}\right|^{-\frac{2 \gamma}{6-5 \gamma}}$ & 1 & 2 & None & Timelike & 0 \\
\hline
\end{tabular}

cally Friedmann and asymptotically quasi-Friedmann solutions. Their description of the asymptotic-KantowskiSachs solutions is also somewhat different: because $r$ is taken to be positive, they do not extend this solution from $z>0$ to $z<0$, so they do not notice these solutions are related to wormholes (cf. Paper II).

CC derive the asymptotically constant-velocity solutions, as well as deriving and plotting Eq. (4.48), but they describe these as "asymptotically Minkowski" and do fully understand their physical interpretation. They also identify the asymptotic solutions which represent a central singularity (i.e. in which $V \rightarrow 1$ at finite $z$ ) but they only include them for $2 / 3<\gamma<1$ and omit them for $0<\gamma<2 / 3$. They also make no distinction between the positive and negative mass singularities. They claim to find another small-distance solution for $2 / 3<\gamma<1$, which they label "Y", but we do not find this and it is probably non-physical.

Analogue of Tables I and II appears in ref. 7] but no analysis of the global structure of the solutions is included, which is another reason why the wormhole connection is missed. Carr and Gundlach 22] have analyzed the global structure of some of these solutions but there is a mistake in the asymptotically Kantowski-Sachs solutions shown in Fig. 15, where $z=\infty$ is wrongly identified with the singularity.
[1] B. J. Carr and A. A. Coley, Class. Quant. Grav. 16, R31 (1999).

[2] B.J. Carr and A. A. Coley, Gen. Rel. Grav. 37, 2165 (2005).

[3] M. E. Cahill and A. H. Taub, Commun. Math. Phys. 21, 1 (1971).

[4] B. J. Carr and A. A. Coley, Phys. Rev. D62, 044023 (2000).

[5] M. Goliath, U. S. Nilsson and C. Uggla, Class. Quant. Grav. 15, 167; ibid, 2841 (1998).

[6] B. J. Carr, A. A. Coley, M. Goliath, U.S. Nilsson and C. Uggla, Class. Quant. Grav. 18, 303 (2001).

[7] B. J. Carr and A. A. Coley, Class. Quant. Grav. 17, 4339 (2000).

[8] A. Ori and T. Piran, Phys. Rev. Lett. 59, 2137 (1987).

[9] A. Ori and T. Piran, Phys. Rev. D. 42, 1068 (1990).

[10] T. Foglizzo and R. N. Henriksen, Phys. Rev. D. 48, 4645 (1993).

[11] T. Harada and H. Maeda, Phys. Rev. D63, 084022 (2001).

[12] C. R. Evans and J. S. Coleman, Phys. Rev. Lett. 72 , 1782 (1994).

[13] H. Maeda, T. Harada and B. J. Carr, arXiv:0707.0530 [gr-qc], to appear in Physical Review D (Paper II).

[14] A.D. Linde, Particle Physics and Inflationary Cosmol- ogy (Harwood Academic Publishers, Chur, Switzerland, 1990).

[15] S. Perlmutter et al., Astrophys. J. 517, 565 (1999); A.G. Riess et al., Astron. J. 116, 1009 (1998); Astron. J. 117, 707 (1999).

[16] T. Harada, H. Maeda, B.J. Carr, Phys. Rev. D74, 024024 (2006).

[17] B. J. Carr and S. W. Hawking, Mon. Not. R. Astron. Soc. 168, 399 (1974).

[18] G. V. Bicknell and R. N. Henriksen, Astrophys. J. 219, 1043 (1978).

[19] B. J. Carr and A. Yahil, Astrophys. J. 360, 330 (1990).

[20] H. Maeda, J. Koga and K-i. Maeda, Phys. Rev. D66, 087501 (2002).

[21] R. Kantowski and R.K. Sachs, J. Math. Phys. 7, 443 (1966).

[22] B. J. Carr and C. Gundlach, Phys. Rev. D67, 024035 (2003).

[23] B.J. Carr, Phys. Rev. D62, 044022 (2000).

[24] H. Maeda and T. Harada, in General Relativity Research Trends. Horizons in World Physics, Volume 249, p.123, ed. R. Albert (Nova Science Publishers, New York, 2006). Preprint gr-qc/0405113

[25] B. J. Carr and A. Koutras, Astrophys. J. 405, 34 (1993).

[26] A. Nusser, Mon. Not. Roy. Astron. Soc. 3751106 (2007). 
[27] B.J. Carr, T. Harada, and H. Maeda, in preparation.

[28] A. Vilenkin and E.P.S. Shellard, Cosmic Strings and Other Topological Defects, (Cambridge University Press,
Cambridge, 1994). 